\title{
MicroRNA-101 inhibits cell proliferation and induces apoptosis by targeting EYA1 in breast cancer
}

\author{
HAITAO GUAN, ZHIJUN DAI, YUGUANG MA, ZHONGWEI WANG, XIAOXU LIU and XIJING WANG
}

Department of Oncology, The Second Affiliated Hospital of Xi'an Jiaotong University, Xi'an, Shaanxi 710004, P.R. China

Received August 4, 2015; Accepted March 22, 2016

DOI: $10.3892 / \mathrm{ijmm} .2016 .2557$

\begin{abstract}
MicroRNAs (miRNAs or miRs) regulate gene expression by negatively modulating the stability or translational efficiency of their target genes by targeting the 3'-untranslated region (3'-UTR). Aberrant miRNA expression has been reported in various types of cancer; miRNAs can function as either oncogenes or tumor suppressor genes in cancer. In this study, we examined the expression level of miR-101 in breast cancer tissues and cell lines by RT-qPCR, and found that miR-101 expression was downregulated in breast cancer tissues and cell lines; indeed, in 6 of the 28 tissue samples, miR-101 could not be detected. Furthermore, miR-101, when transfected into SKBR3 cells, inhibited cell proliferation and promoted apoptosis, while miR-101 inhibitor had the opposite effect. A dual-luciferase reporter assay revealed that miR-101 targeted the 3'-UTR of eyes absent homolog 1 (Drosophila) (EYA1). Western blot analysis demonstrated a significantly decreased protein level of EYA1 in the SKBR3 cells transfected with miR-101 mimic, whereas transfection with miR-101 inhibitor led to an increased level of EYA1. Moreover, an increased expression of EYA1 was also found in breast cancer tissues and cell lines. The silencing of EYA1 using siRNA targeting EYA1 (EYA1-siRNA) significantly inhibited SKBR3 cell proliferation and promoted apoptosis, and also suppressed the increased proliferation induced by transfection with miR-101 inhibitor. The protein expression levels of Notch signaling components (jagged1, Hes1 and Hey1) were significantly decreased by transfection with miR-101 mimic and EYA1siRNA, and were increased by transfection with miR-101 inhibitor. Furthermore, the elevated protein expression levels of jagged1, Hes1 and Hey1 induced by transfection with miR-101
\end{abstract}

Correspondence to: Dr Haitao Guan, Department of Oncology, The Second Affiliated Hospital of Xi'an Jiaotong University, 157 Xiwu Road, Xi'an, Shaanxi 710004, P.R. China

E-mail: haitaoguancn@163.com

Abbreviations: miRNA or miR, microRNA; 3'-UTR, 3'-untranslated region; EYA1, eyes absent homolog 1 (Drosophila)

Key words: breast cancer, microRNA-101, eyes absent homolog 1 (Drosophila), proliferation, apoptosis inhibitor in the SKBR3 cells were significantly decreased by transfection with EYA1-siRNA. Taken together, these results suggest that miR-101 is downregulated in breast cancer, and can inhibit cell proliferation and promote apoptosis by targeting EYA1 through the Notch signaling pathway.

\section{Introduction}

Breast cancer is the most frequently diagnosed type of cancer and the leading cause of cancer-related mortality in women worldwide, accounting for 1.7 million cases and 521,900 deaths in 2012 (1). Although methods for the screening and treatment of breast cancer have improved over the years (2), the molecular pathogenesis of breast cancer remains unclear and breast cancer remains a major public health challenge (3).

MicroRNAs (miRNAs or miRs) are small non-coding RNA molecules that modulate gene expression by negatively regulating the stability or translational efficiency of their target mRNAs by targeting the 3'-untranslated region (3'-UTR) (4). More than 474 miRNA genes have been identified in the human genome, and over $10 \%$ of the protein-coding mRNAs may be conserved targets of miRNAs $(5,6)$, which suggests an important role of miRNAs in biological processes in humans. It has been shown that miRNAs are aberrantly expressed or mutated in cancer, and the analysis of miRNA profiles can help to distinguish between normal and cancerous tissue (7). The majority of miRNAs, such as miR-21 and miR-155, are overexpressed in several human cancers (8-11), while other miRNAs, such as Let-7 and miR-34, are frequently downregulated in human cancers (12-15); thus, miRNAs can function as either oncogenes or tumor suppressor genes (16). Various cellular processes, including proliferation, apoptosis, cell cycle progression, differentiation and metastasis, have been implicated to be under the regulation of miRNAs (17-19). All these abovementioned studies suggest an important role of miRNAs in the development of cancer. A complete understanding of miRNAs and cancer may provide new insight into the molecular mechanisms underlying tumorigenesis.

Breast cancer was one of the first solid tumors in which miRNA expression was profiled. Iorio et al (20) identified 29 miRNAs whose expression was significantly dysregulated in breast cancer compared to normal breast tissues, including miR-10b and miR-125b, which were downregulated, and miR-145 and miR-21, which were upregulated in breast cancer. 
miR-101 was also downregulated in breast cancer (20), and its established targets include enhancer of zeste homolog 2 (EZH2), DNA methyltransferase 3A (DNMT3A), and microphthalmiaassociated transcription factor (MITF) (21-23). However, there are only a few studies available on the potential function of miR-101 in breast cancer $(20,41)$.

The eyes absent (Drosophila) (EYA) proteins are crucial regulators in the development of the eye $(24,25)$. Four homologs of the EYA family proteins (EYA 1-4) are defined by a conserved 275-amino acid carboxyl-terminal motif, referred to as the EYA domain (ED). The overexpression of EYA family proteins has been reported in many cancer cell lines, including in ovarian and breast cancer cell lines $(26,27)$, and it has also been reported that the overexpression of EYA proteins promotes cell proliferation through the epidermal growth factor receptor (EGFR)/RAS/ mitogen-activated protein kinase (MAPK) and Notch signaling pathways $(28,29)$. EYA1 is an essential transactivated gene whose mutation can cause branchio-oto-renal and branchio-oto syndromes. The overexpression of EYA1 has been observed in cancers $(30,31)$, and EYA1 can form a transcription complex with sine oculis homeobox 1 (SIX 1), which regulates expression of a number of downstream target genes that are important for cell proliferation, survival and migration (32). Although EYA1 has been shown to play an important role in cancer development and progression, whether miRNAs can regulate EYA1 expression has not yet been reported, at least to the best of our knowledge. Thus, our study aimed to assess the expression level of miR-101 and to elucidate the regulatory effects of miR-101 in breast cancer.

\section{Materials and methods}

Human tissue specimens. Breast cancer and normal adjacent breast tissues were obtained from 28 patients with breast cancer who had not received any pre-operative cancer treatments at the Second Affiliated Hospital of Xi'an Jiaotong University, Xi'an, China. The tissue samples obtained from surgery were frozen in liquid nitrogen immediately. Informed consent was obtained from all patients. This study was approved by the Ethics Committee of the Second Affiliated Hospital of Xi'an Jiaotong University.

Cell culture. The breast cancer cell lines, MDA-MB-231, T-47D, SKBR3, MCF-7, BT474 and HS578T, and the normal breast epithelial cell line, MCF-10, were all obtained from ATCC (Manassas, VA, USA) and were cultured in Dulbecco's modified Eagle's medium (Invitrogen, Carlsbad, CA, USA) containing 1\% penicillin/streptomycin (Sigma, St. Louis, MO, USA) and supplemented with $10 \%$ fetal bovine serum (Invitrogen) in a $5 \% \mathrm{CO}_{2}$ cell culture incubator.

miRNA transfection and small RNA interference. The SKBR3 cells were seeded in 6-well plates, and miR-101 mimic (miR-101) or its negative control (NC-miR), and miR-101 inhibitor (miR-101-in) or its negative control (miR-NC-in) (Dharmacon, Lafayette, CO, USA), were transfected into the cells using Lipofectamine 2000 transfection reagent (Invitrogen) according to the instructions provided by the manufacturer. The cells were harvested for analysis after being incubated for $48 \mathrm{~h}$.
To knockdown EYA1 expression, siRNA targeting EYA1 (EYA1-siRNA) and its negative control (EYA1-NC) (Santa Cruz Biotechnology, Santa Cruz, CA, USA) were transfected into the SKBR3 cells. Following culture for $48 \mathrm{~h}$, the cells were harvested and the expression level of miR-101 and EYA1 was measured as described below.

Cell proliferation assay. Cell proliferation was determined using CCK-8 reagent (Beyotime, Nantong, China) according to the manufacturer's instructions. The cells were plated into a 96-well plate at $5 \times 10^{4}$ cells/well. After 1, 2, 3, 4 and 5 days, the cells were treated with CCK- 8 reagent and incubated for $2 \mathrm{~h}$. The absorbance was measured using a Bio-Rad microplate reader (Bio-Rad, Hercules, CA, USA) at a wavelength of $450 \mathrm{~nm}$.

Apoptosis assay. Apoptotic cells were detected using the Annexin V-FITC/PI apoptosis detection kit (Abcam, Cambridge, UK). In brief, $1 \times 10^{5}$ cells were harvested and incubated with Annexin V-FITC for $5 \mathrm{~min}$ in the dark, and then stained with PI for a further $5 \mathrm{~min}$. The apoptotic cells were analyzed using a FACSCalibur flow cytometer (Becton Dickinson, San Jose, CA, USA).

Reverse transcription-quantitative PCR $(R T-q P C R)$. To determine the abundance of miR-101, total miRNAs were extracted from the SKBR3 cells using the miRcute miRNA isolation kit (Tiangen, Beijing, China), and cDNA was synthesized using the miRcute miRNA First-Strand cDNA synthesis kit (Tiangen). qPCR was performed using the miScript SYBR Green PCR kit (Qiagen, Valencia, CA, USA) according to the manufacturers instructions. U6 was used as a quantitative and qualitative control to normalize miRNA expression. The RT-PCR primers for miR-101 were forward, 5'-TGGGCTACAGTACT GTGATA-3' and reverse, 5'-TGCGTGTCGTGGAGTC-3' as previously described (36).

To determine the mRNA expression level of EYA1, total RNA was extracted from the SKBR3 cells using TRIzol reagent (Invitrogen), and cDNA was synthesized using MMLV reverse transcriptase (Clontech, Palo Alto, CA, USA). qPCR was performed using TaqMan PCR Master Mix and an ICycler system (Beijing Solarbio Science \& Technology Co., Ltd., Beijing, China). $\beta$-actin was used as a negative control. The RT-PCR primers for EYA1 [as previously described (33)] were forward, 5'-TAACGGACAGGACCTAAGCA-3' and reverse, 5'-TTTCTCATCCAgTCCACACC-3'.

Target prediction. To determine whether EYA1 is a direct tareget of miR-101, we searched for the potential targets of miR-101 using the prediction programs, microRNA.org (http:// www.microrna.org/microrna/home.do), TargetScan (http:// www.targetscan.org/vert_60/) and PicTar (http://www.pictar. org/cgi-bin/PicTar_vertebrate.cgi).

Dual-luciferase reporter assay. The SKBR3 cells co-transfected with miR-101 mimic and luciferase reporter constructs containing a wild-type or mutant EYA1 3'-UTR (Promega, Madison, WI, USA) were seeded into a 24-well plate. Forty-eight hours later, the cells were harvested and the luciferase activity was analyzed using the dual-luciferase reporter assay kit (Promega). All transfection experiments were reproduced at least 3 times. 
A

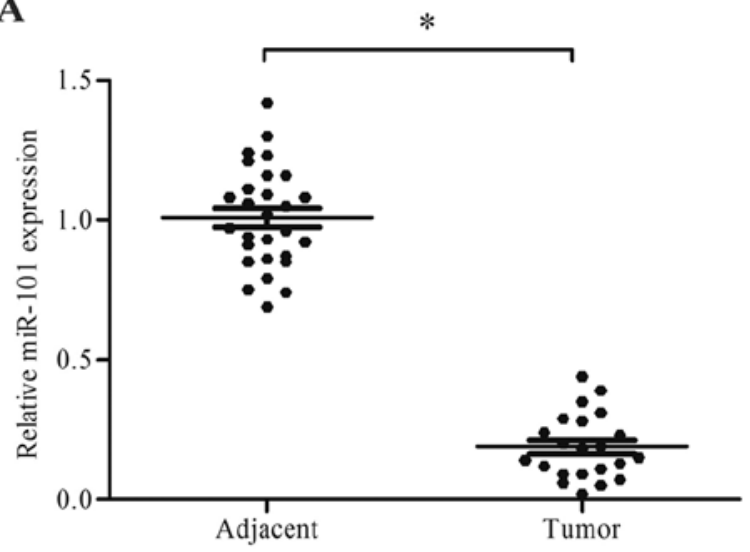

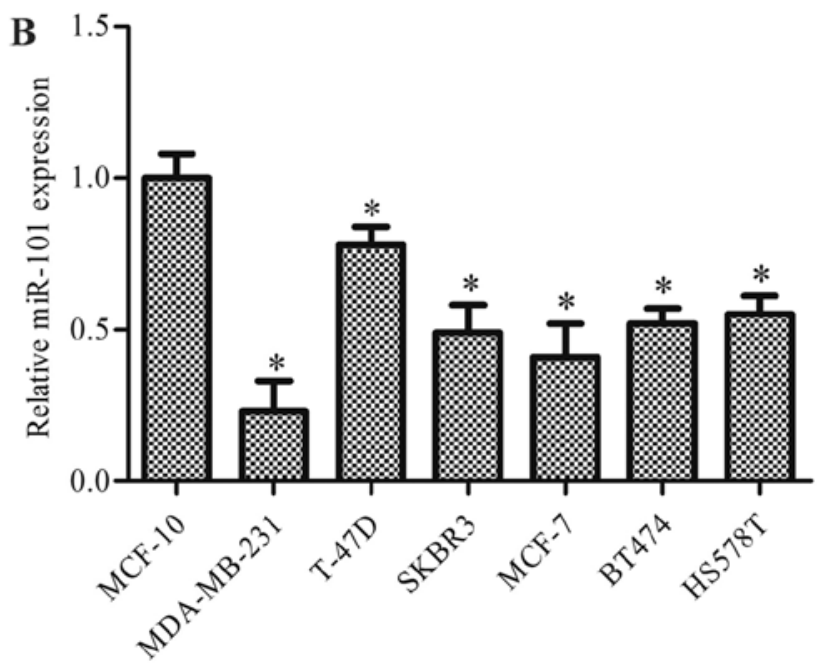

Figure 1. miR-101 expression is downregulated in breast cancer tissues and cell lines. (A) Breast cancer tissues were obtained from breast cancer patients and frozen in liquid nitrogen immediately. An RT-qPCR assay was used to measured miR-101 expression. $\mathrm{n}=28$ samples; ${ }^{*} \mathrm{p}<0.05$ vs. adjacent normal tissue. (B) Six breast cancer cell lines and the MCF-10 breast epithelial cell line were cultured in Dulbecco's modified Eagle's medium, and miR-101 expression was analyzed by RT-qPCR. $n=3$ experiments, ${ }^{*} \mathrm{p}<0.05$ vs. MCF-10 cells.

Western blot analysis. The SKBR3 cells were lysed with RIPA buffer (Beyotime, Bejing, China) to extract total protein, and the protein concentration was quantified using the Bradford assay (Beyotime). A total of $20 \mu \mathrm{g}$ protein was separated by electrophoresis on a $12 \%$ SDS-PAGE gel. Proteins were transferred onto nitrocellulose membranes (Bio-Rad), and then incubated with $2 \%$ non-fat milk to block non-specific binding for $1 \mathrm{~h}$ at $37^{\circ} \mathrm{C}$. The membranes were then incubated with the following primary antibodies: rabbit anti-EYA1 (ab85009), rabbit anti-jagged1 (ab7771), rabbit anti-Hey1 (ab22614) and rabbit anti-Hes1 (ab71559) polyclonal antibodies (Abcam) overnight at $4^{\circ} \mathrm{C}$. The membranes were washed with TAS-Tween (TBST) 3 times, and then incubated with secondary antibody horseradish peroxidase (HRP)-conjugated goat anti-rabbit IgG (ab6762; Abcam) for $4 \mathrm{~h}$ at $37^{\circ} \mathrm{C}$. Proteins were detected using an enhanced chemiluminescence detection system (Amersham, Piscataway, NJ, USA) after washing with TBST 3 times.

Statistical analysis. Data are presented as the means \pm standard deviation (SD) and the statistically significant differences between different groups were analyzed using SPSS 18.0 software (SPSS nc., Chicago, IL, USA) and the Student's t-test. A value of $\mathrm{p}<0.05$ was considered to indicate a statistically significant difference.

\section{Results}

miR-101 is downregulated in breast cancer tissues and cell lines. The results of RT-qPCR revealed that miR-101 was significantly downregulated in the breast cancer tissues compared with the normal adjacent breast tissues, and miR-101 could not be detected in 6 of the 28 breast cancer tissues (Fig. 1A, p<0.05). The expression of miR-101 was also examined in 6 breast cancer cell lines, and the results revealed that miR-101 was markedly downregulated in all 6 breast cancer cell lines compared with the normal breast epithelial cell line, MCF-10 (Fig. 1B, p<0.05).
miR-101 regulates cell proliferation and apoptosis. The downregulation or even the loss of miR-101 in breast cancer suggested that it may play a role in the development and progression of breast cancer. To determine the role of miR-101 in breast cancer, we transfected miR-101, miR-101-in or their negative control into the SKBR3 cells, and examined the effects of miR-101 on breast cancer cell proliferation by CCK- 8 assay. The results revealed that transfection with miR-101 significantly upregulated miR-101 expression, whereas transfection with miR-101-in significantly downregulated miR-101 expression (Fig. 2A, p<0.05). The overexpression of miR-101 significantly decreased the proliferation of the SKBR3 cells, and the decreased expression of miR-101 promoted the proliferation of the SKBR3 cells in a timedependent manner (Fig. 2B, p<0.05). To determine whether miR-101 also regulates the apoptosis of breast cancer cells, we examined the apoptosis of the SKBR3 cells transfected with miR-101 or miR-101-in using an Annexin V-FITC assay. A marked increase in apoptosis was induced by transfection with miR-101 mimic in the SKBR3 cells (Fig. 2C, p<0.05).

miR-101 directly targets EYA1 in breast cancer cells. To elucidate the potential molecular mechanisms responsible for the inhibitory effects of miR-101 on the proliferation of breast cancer cells and its apoptosis promoting effects, we searched for the potential targets of miR-101 using the prediction programs microRNA.org, TargetScan and PicTar. Two conserved pairing target regions (position 198-204 and 1899-1906) between the EYA1 3'-UTR and miR-101 were found, and according to the context++ score, position 1899-1906 was selected for further analysis (Fig. 3A), suggesting that EYA1 may be a potential target of miR-101. To determine whether miR-101 directly targets the 3'-UTR region of EYA1, a dual-luciferase reporter assay was performed. Transfection with miR-101 significantly reduced the luciferase reporter activity of the wild-type EYA1 3'-UTR compared with the luciferase reporter activity of the mutant 3'-UTR. Conversely, miR-101 caused no significant changes in 
A

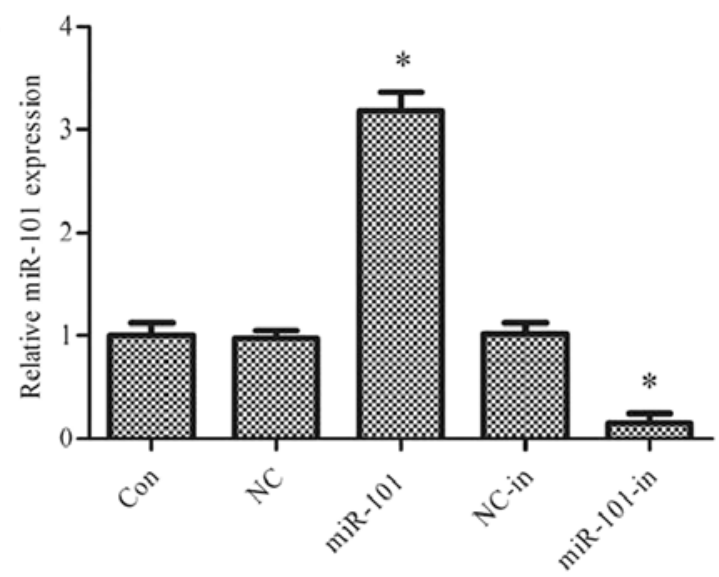

B

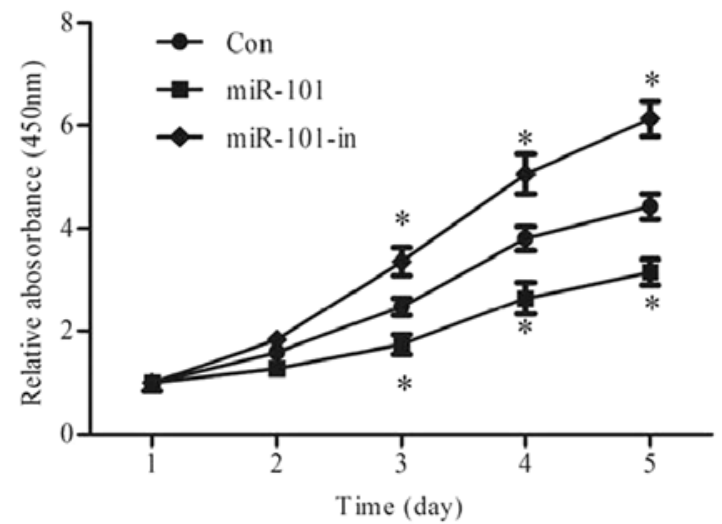

C
Con

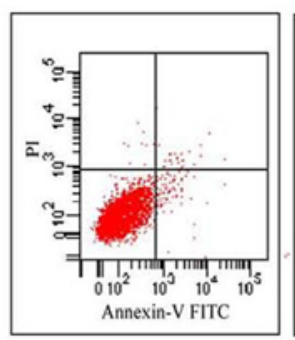

$\operatorname{miR}-101$

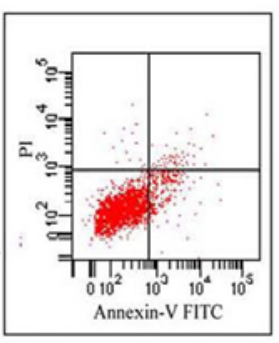

miR-101-in

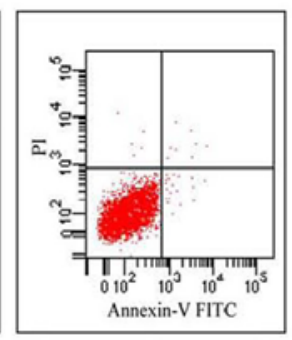

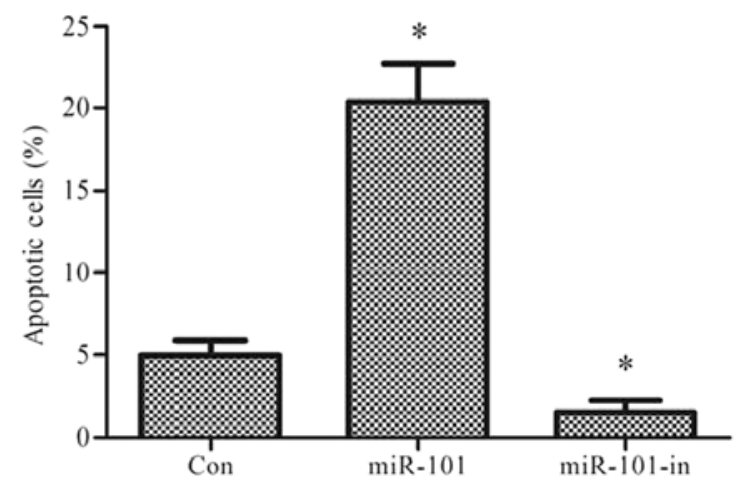

Figure 2. miR-101 regulates SKBR3 cell proliferation and apoptosis. (A) SKBR3 cells were transfected with miR-101 mimic (miR-101) or its negative control (NC), and inhibitor miR-101 (miR-101-in) or its negative control (NC-in). miR-101 expression was examined by RT-qPCR. n=3 experiments; ${ }^{*}$ p $<0.05$ vs. control (untransfected cells). (B) SKBR3 cells were transfected with miR-101 or miR-101-in, and cell proliferation was measured at different time points (1,2,3,4 and 5 days) using a CCK-8 assay. $n=3$ experiments; ${ }^{*}$ p $<0.05$ vs. control at the corresponding time point. (C) SKBR3 cells were transfected with miR-101 or miR-101-in, and apoptosis was determined using the Annexin V-FITC/PI apoptosis detection kit. $\mathrm{n}=3$ experiments; ${ }^{*} \mathrm{p}<0.05$ vs. control (untransfected cells).

the expression of the transcript containing the mutant 3'-UTR of EYA1. The luciferase activity of the reporter plasmid was also not affected by transfection with miR-101 inhibitor (Fig. 3B).

As miR-101 suppressed the luciferase transcript containing the wild-type EYA1 3'-UTR, we speculated that miR-101 may regulate the expression of EYA1 in breast cancer. To verify this, we examined the potential regulatory effect of miR-101 on the expression of EYA1. miR-101 or miR-101-in was transfected into the SKBR3 cells. Western blot analysis revealed a significantly decreased protein level of EYA1 in the SKBR3 cells transfected with miR-101 compared with the controls (Fig. 3C, p<0.05).

EYA1 is overexpressed in breast cancer tissues and cell lines. As EYA1 was found to be a direct target of miR-101 and miR-101 expression was found to be downregulated in breast cancer, we examined the expression level of EYA1 in the breast cancer tissues and cell lines. The expression of EYA1 was significantly higher in the breast cancer tissues than the adjacent normal breast tissues (Fig. 4A, p<0.05). RT-qPCR analysis of the breast cancer cell lines also revealed that EYA1 expression in the breast cancer cell lines, apart from the T-47D cell line, was significantly upregulated (Fig. 4B, p<0.05).
EYAl is involved in the regulation of cell proliferation and apoptosis by $m i R-101$. EYA1 is a crucial regulator of cell growth in cancers (31). Our study explored the role of EYA1 in the regulatory effects of miR-101 in breast cancer. The expression of EYA1 was silenced using EYA1siRNA ( $<<0.05$, Fig. 5A). CCK-8 assay was then performed to examine the proliferation of the SKBR3 cells transfected with miR-101, miR-101-in, or EYA1-siRNA, or co-transfected with EYA1-siRNA and miR-101 inhibitor. The inhibitor of miR-101 (miR-101-in) promoted cell proliferation. When the cells were transfected with miR-101 or EYA1-siRNA, cell proliferation decreased markedly $(p<0.05$, Fig. $5 B)$. Transfection with EYA1-siRNA also significantly inhibited the increased proliferation and decreased the apoptosis that was induced by the miR-101-inhibitor. The Annexin V-FITC assay indicated that miR-101 promoted apoptosis and that miR-101-in inhibited apoptosis, whereas EYA1-siRNA and co-transfection with EYA1-siRNA and miR-101-in promoted apoptosis (p<0.05, Fig. 5C).

miR-101 regulates the Notch signaling pathway through EYA1. To determine the signaling pathway involved in the regulatory effects of miR-101 in breast cancer, we analyzed the Notch 
A

EYA1-3'-UTR 5'-AAAUUUAUUUGUGAUGUACUGUA-3' (wild-type)

hsa-miR-101 3'-AGUCAAUAGUGU-------CAUGACAU-5'

EYA1-3'-UTR 5'-AAAUUUAUUUGUGAUCTUGACTA-3' (mutant)
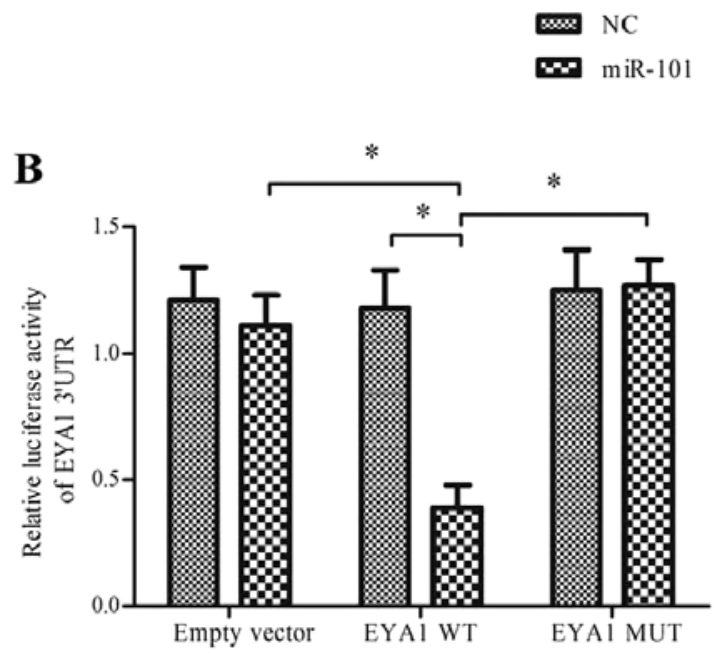

C
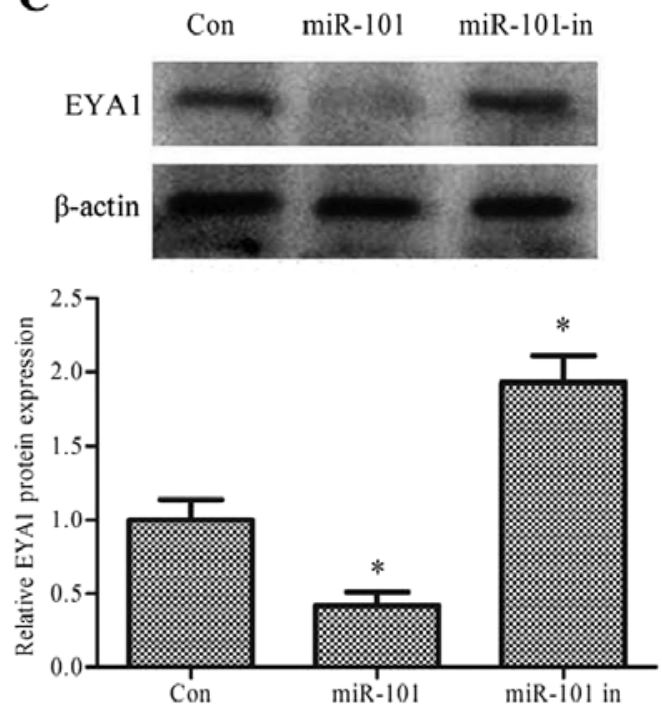

Figure 3. miR-101 directly downregulates the expression of EYA1. (A) The predicted miR-101 target sequence in the wild-type EYA1 3'-UTR and a similar sequence containing mutations (EYA1-3'-UTR). (B) Dual-luciferase assay of SKBR3 cells co-transfected with miR-101 and luciferase reporter constructs containing wild-type or mutant EYA1 3'-UTR. (C) Western blot analysis of EYA1 expression in SKBR3 cells transfected with miR-101 or miR-101-in. $\mathrm{n}=3$ experiments; ${ }^{*} \mathrm{p}<0.05$ vs. control (untransfected cells).
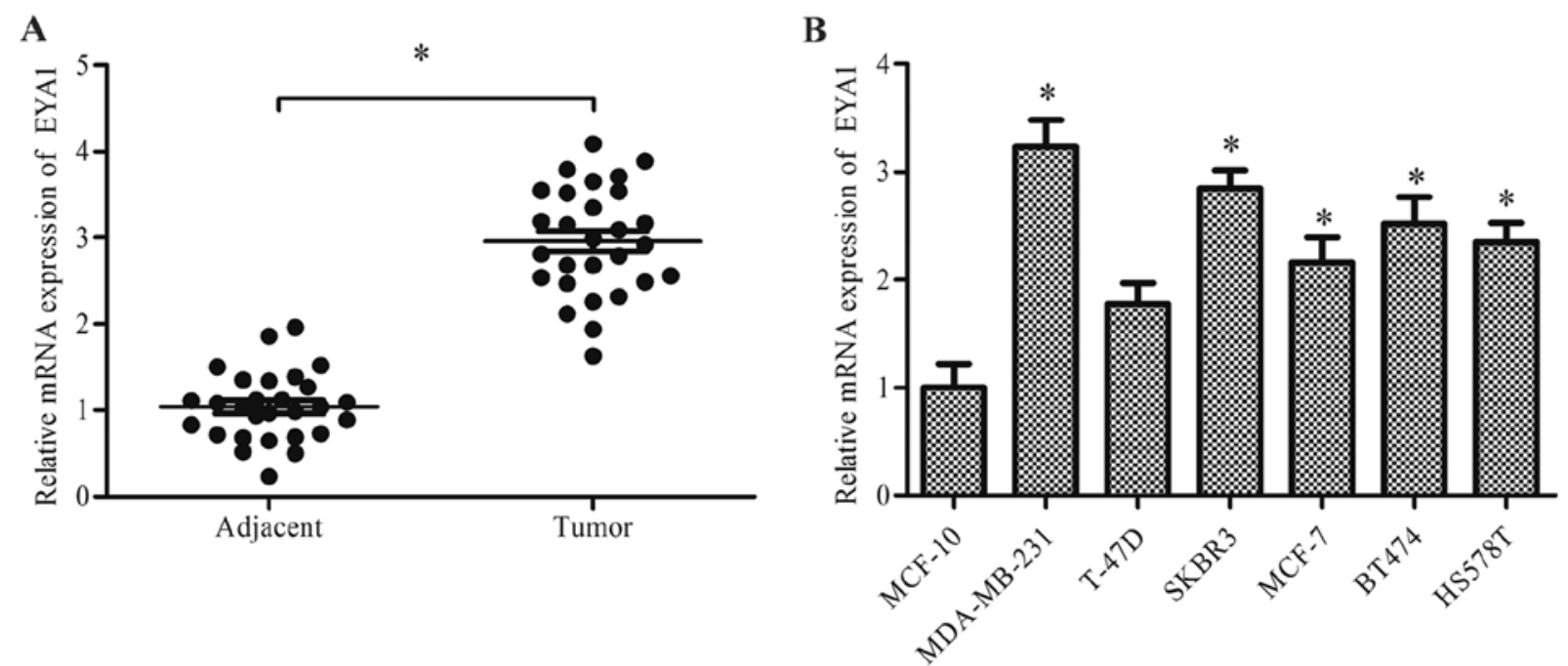

Figure 4. EYA1 is overexpressed in breast cancer tissues and cell lines. (A) Breast cancer tissues were obtained from breast cancer patients and frozen in liquid nitrogen immediately. An RT-qPCR assay was used to measured EYA1 expression. n=28 samples; * $\mathrm{p}<0.05$ vs. adjacent normal tissue. (B) Six breast cancer cell lines and the breast epithelial cell line MCF-10 were cultured in Dulbecco's modified Eagle's medium and the EYA1 expression was analyzed by RT-qPCR. $\mathrm{n}=3$ experiments; ${ }^{\mathrm{p}}<0.05$ vs. MCF-10 cells.

signaling pathway. The expression level of jagged1, one of the important ligands of Notch, and Hes1 and Hey1, the downstream genes of the Notch pathway, were analyzed in SKBR3 cells transfected with miR-101, miR-101-in or EYA1-siRNA. The protein expression levels of jagged1, Hes1 and Hey1 were significantly decreased following transfection with miR-101 and EYA1-siRNA, but were increased following transfection with miR-10-in $(\mathrm{p}<0.05)$. Furthermore, the elevated protein expression levels of jagged1, Hes1 and Hey1 induced by miR-101-in in the SKBR3 cells were significantly decreased by transfection with EYA1-siRNA (p<0.05, Fig. 6).

\section{Discussion}

The dysregulation of miRNAs has frequently been reported in several types of cancer (34). Increasing evidence has 

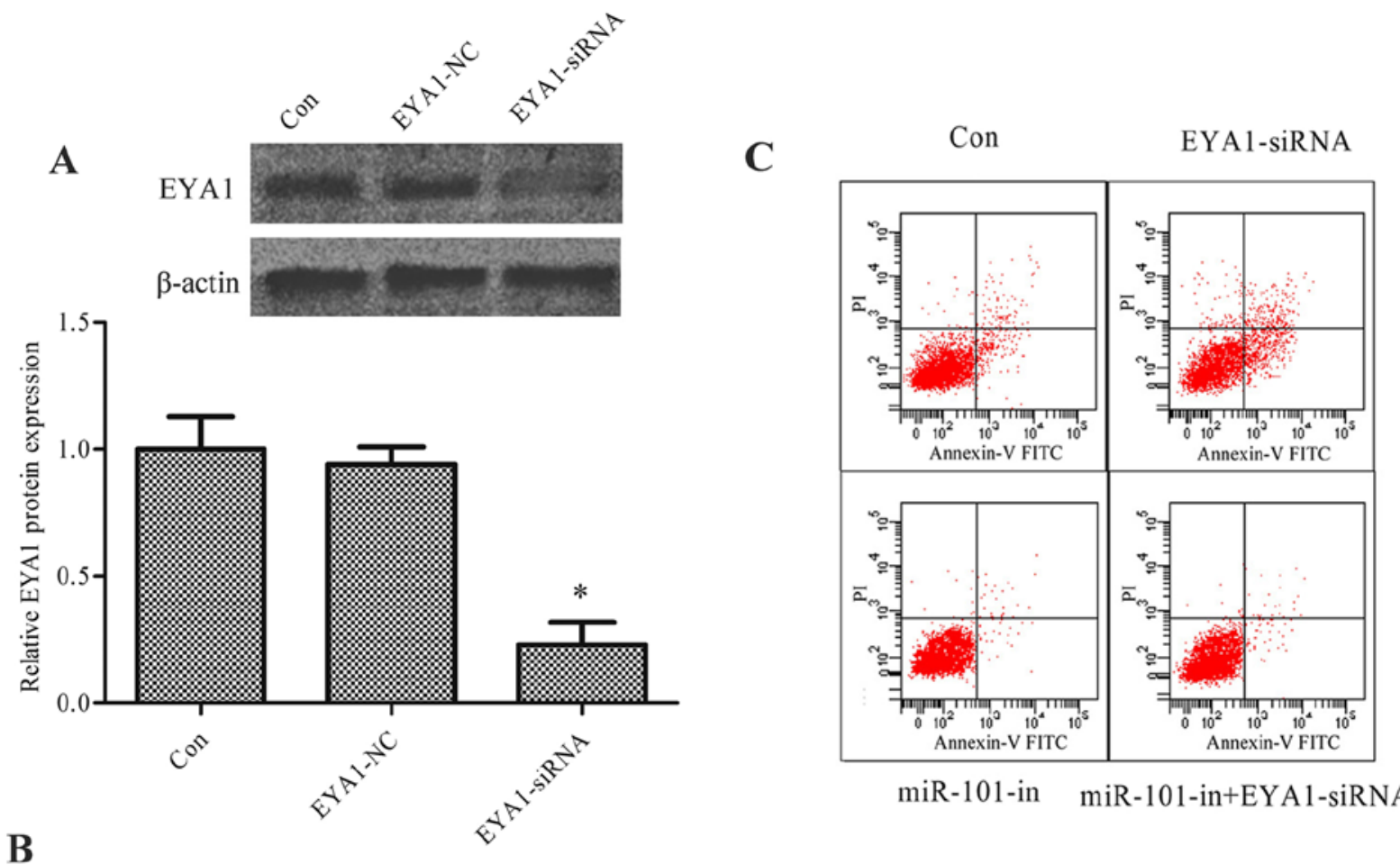

B
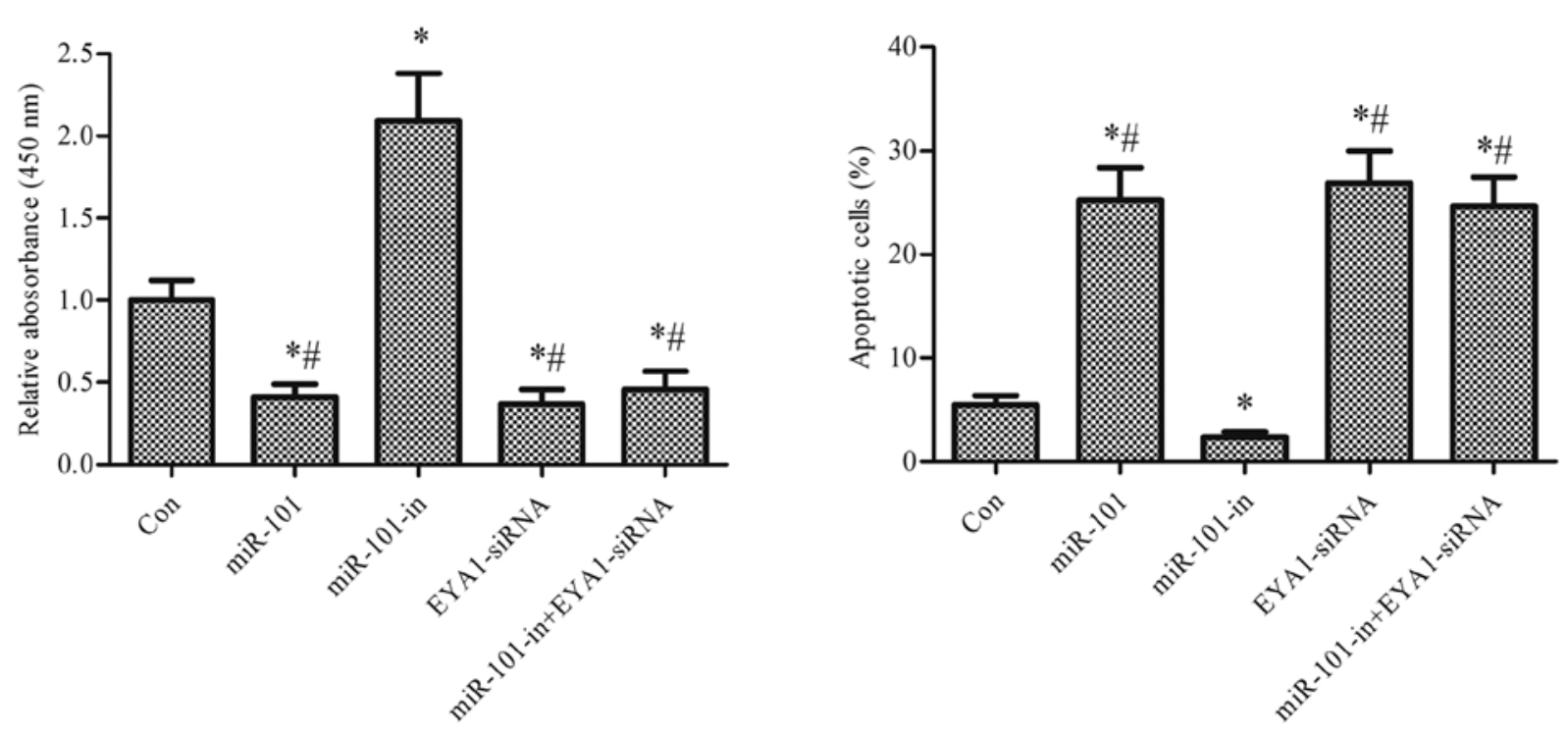

Figure 5. EYA1 is involved in the regulation of cell proliferation and apoptosis by miR-101. (A) SKBR3 cells were transfected with miR-101, miR-101-in, or EYA-1 siRNA, or co-transfected with miR-101-in and EYA1-siRNA. (C) Cell proliferation was detected by CCK-8 assay. (B) SKBR3 cells were transfected with miR-101, miR-101-in, or EYA-1 siRNA, or co-transfected with miR-101-in and EYA1-siRNA. Apoptosis was examined using the Annexin V-FITC/PI apoptosis detection kit. $\mathrm{n}=3$ experiments; ${ }^{\mathrm{p}} \mathrm{p}<0.05$ vs. control (untransfected cells), ${ }^{*} \mathrm{p}<0.05$ vs. transfection with miR-101-in.

demonstrated a cancerocidal or carcinogenic role of miRNAs in mediating the process of tumorigenesis and the behavior of cancer cells (35). In the present study, we confirmed that miR-101 expression was downregulated or even lost in human cancer tissues and cell lines. We also found that miR-101 inhibited cell growth and promoted apoptosis in vitro. We further demonstrated that EYA1 is a functional target of miR-101, and that miR-101 regulates cell behavior by targeting EYA1 through the Notch signaling pathway.

miRNA-101 has been shown to be downregulated in gastric cancer, glioblastoma, prostate cancer, colon cancer and hepatocellular carcinoma, and is involved in cell migration, invasion, proliferation and apoptosis (36-40). The decreased expression of miR-101 has also been detected in human breast cancer tissues (20). In this study, we detected the expression of miR-101 in human breast cancer tissues and cell lines, and found that miR-101 was downregulated in both tissues and cell lines compared with adjacent normal tissues and cells, respectively. Indeed, the expression of miR-101 was not detectable in 6 of the 28 breast cancer tissues, which is in accordance with the study by Varambally et al (41), who found that 6 of 29 breast cancer tissues were negative for miR-101. Thus, we speculated that miR-101 may function as a tumor suppressor gene in breast cancer. To verify this, we examined cell prolif- 
A

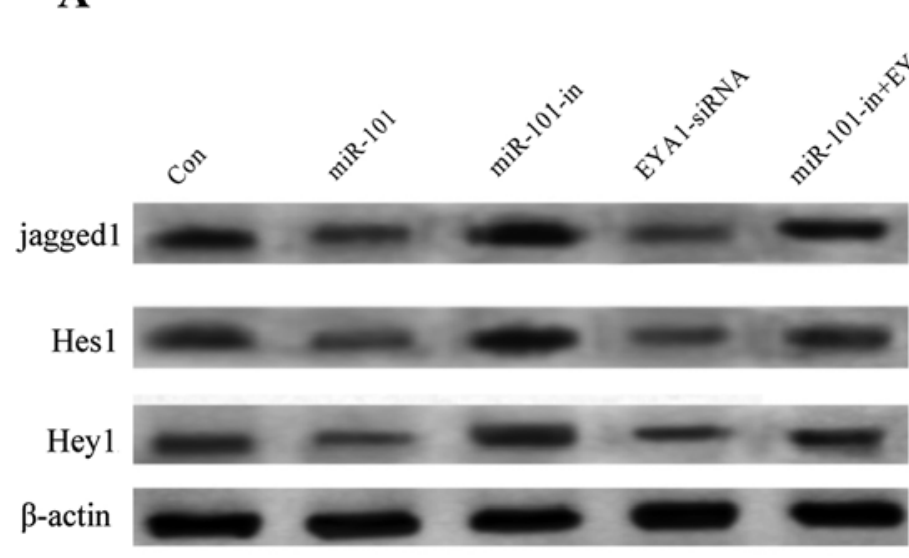

C

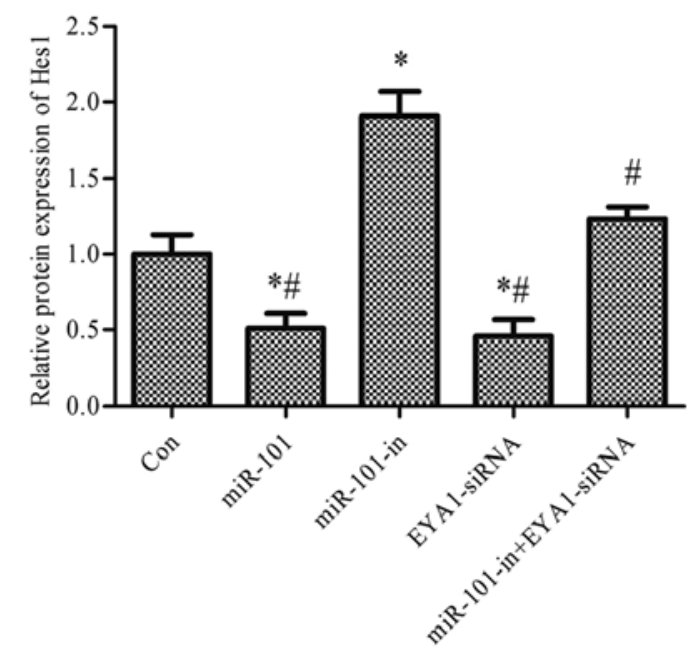

B
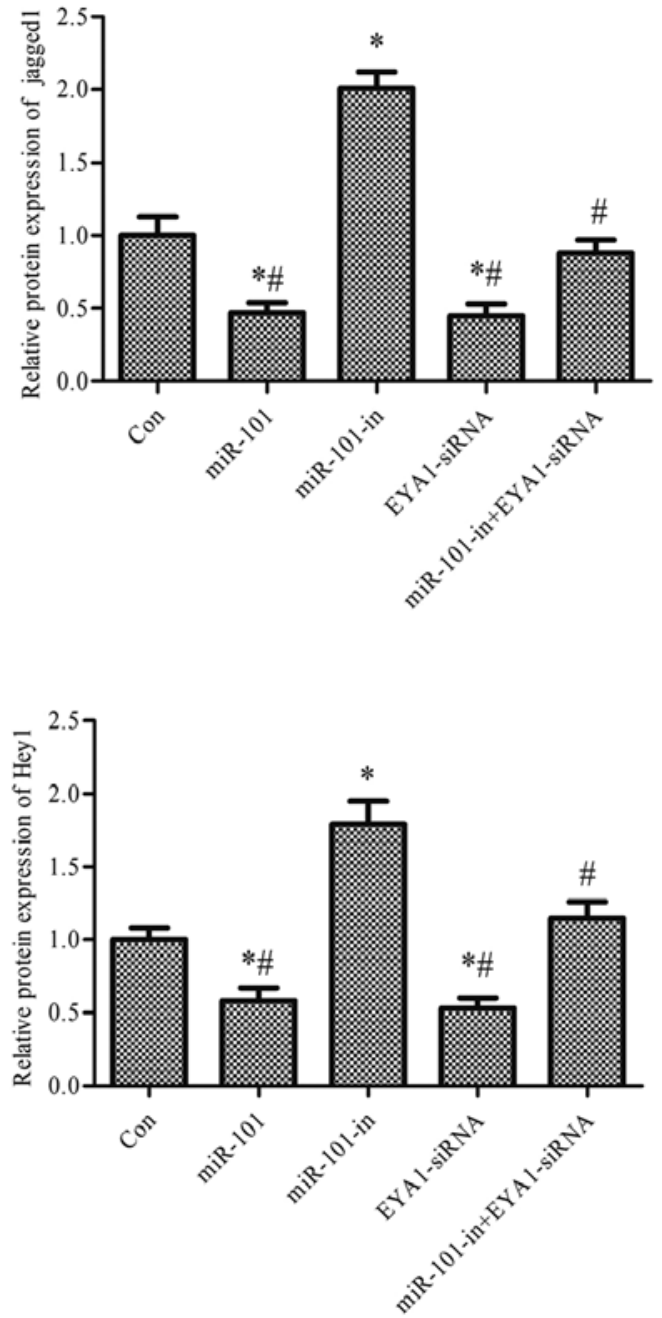

Figure 6. miR-101 regulates the Notch signaling pathway via EYA1. (A) Representative western blot showing the expression of jagged1, Hes1 and Hey1 in all groups, and a column diagram showing the quantification of (B) jagged1, (C) Hes1 and (D) Heyl. The column indicates thye means \pm SEM. ${ }^{*}<<0.05$ vs. control (untransfected cells), ${ }^{\#} \mathrm{p}<0.05$ vs. transfection with miR-101-in.

eration and apoptosis following the up- or downregulation of miR-101 by transfecting the cells with miR-101 or miR-101-in, respectively. The overexpression of miR-101 inhibited cell proliferation and promoted apoptosis, whereas the downregulation of miR-101 exerted an opposite effect, indicating that miR-101 may serve as a novel therapeutic marker in breast cancer patients.

Cancer is a complex disease that is strongly dependent on the regulation of gene expression. mRNAs exert their regulatory effects by base-pairing with their target mRNAs, and a single miRNA can regulate multiple downstream target mRNAs. Although proto-oncogene MYCN, histone methyltransferase EZH2 and DNM3A have been reported to be targets of miR-101 in cancers $(22,41,42)$, the regulatory effects of miR-101 in cancer are not yet fully understood. Thus, in the present study, we searched for the target gene of miR-101 on microRNA.org, TargetScan and PicTar. Two conserved pairing target regions between the EYA1 3'-UTR and miR-101 were found. Position 1899-1906 was selected for further analysis through luciferase reporter assays. The results confirmed that EYA1 is a direct target of miR-101, and furthermore, miR-101 downregulated the protein expression of
EYA1. EYA1 is an important factor of the retinal determination gene network involved in organismal development (43). The dysregulation of EYA1 has been reported in many types of cancer in recent years $(33,44,45)$; however, the role of EYA1 in tumor growth may vary in different types of cancer. The decreased expression of EYA1 in gastric tumor samples has been described, and EYA1 has been shown to negatively correlate with tumor size, lymphatic invasion and distant metastasis (33).

$\mathrm{Wu}$ et al (31) investigated the expression of EYA1 and showed that it was upregulated both in breast cancer tissues and cell lines, and that EYA1 contributed to cancer cell growth by promoting cell proliferation and DNA synthesis, and also reduced apoptosis. Our study demonstrated that the expression level of EYA1 was significantly upregulated in breast cancer tissues and cells, and that the knockdown of EYA1 inhibited the effects of miR-101 on cell proliferation and apoptosis. Taken together, our study indicates that the regulatory effects of miR-101 on the growth and development of breast cancer may be partly mediated by targeting EYA1.

Notch is one of the fundamental signaling pathways that regulates embryonic cell fate decisions, differentiation, prolif- 
eration and patterning $(46,47)$. The dysregulation of Notch signaling was discovered in various types of cancer and has been shown to correlate with cell cycle progression, proliferation and apoptosis $(48,49)$. The aberrant activation of the Notch pathway leads to adenocarcinoma of the mammary gland (50). Stylianou et al (51) demonstrated that activated Notch signaling protects breast cancer cells from apoptosis. Our study revealed that Notch was the downstream signaling pathway of EYA1, which was under the regulatory effect of miR-101.

In conclusion, our results demonstrate that miR-101 is downregulated both in breast cancer tissues and cell lines, and that it directly downregulates the expression of EYA1 at the post-translational level. The Notch signaling pathway is also proposed to play an important role in the regulatory effects of miR-101. Considering these results, miR-101 may be used as a potential therapeutic marker in breast cancer.

\section{References}

1. Torre LA, Bray F, Siegel RL, Ferlay J, Lortet-Tieulent J and Jemal A: Global cancer statistics, 2012. CA Cancer J Clin 65: 87-108, 2015.

2. Rhodes DJ, O'Connor MK, Phillips SW, Smith RL and Collins DA: Molecular breast imaging: a new technique using technetium Tc $99 \mathrm{~m}$ scintimammography to detect small tumors of the breast. Mayo Clin Proc 80: 24-30, 2005.

3. Gompel A: Breast cancer incidence rates in US women are no longer declining. Climacteric 14: 690-691, 2011.

4. Hwang HW and Mendell JT: MicroRNAs in cell proliferation, cell death, and tumorigenesis. Br J Cancer 96 (Suppl 96) R40-R44, 2007.

5. Griffiths-Jones S, Grocock RJ, van Dongen S, Bateman A and Enright AJ: miRBase: microRNA sequences, targets and gene nomenclature. Nucleic Acids Res 34: D140-D144, 2006.

6. Griffiths-Jones S: The microRNA Registry. Nucleic Acids Res 32: D109-D111, 2004.

7. Blenkiron C, Goldstein LD, Thorne NP, Spiteri I, Chin SF, Dunning MJ, Barbosa-Morais NL, Teschendorff AE, Green AR, Ellis IO, et al: MicroRNA expression profiling of human breast cancer identifies new markers of tumor subtype. Genome Biol 8: R214, 2007.

8. Frezzetti D, De Menna M, Zoppoli P, Guerra C, Ferraro A, Bello AM, De Luca P, Calabrese C, Fusco A, Ceccarelli M, et al Upregulation of miR-21 by Ras in vivo and its role in tumor growth. Oncogene 30: 275-286, 2011.

9. Yan LX, Huang XF, Shao Q, Huang MY, Deng L, Wu QL, Zeng YX and Shao JY: MicroRNA miR-21 overexpression in human breast cancer is associated with advanced clinical stage, lymph node metastasis and patient poor prognosis. RNA 14: 2348-2360, 2008.

10. Yang M, Shen H, Qiu C, Ni Y, Wang L, Dong W, Liao Y and Du J: High expression of miR-21 and miR-155 predicts recurrence and unfavourable survival in non-small cell lung cancer. Eur J Cancer 49: 604-615, 2013.

11. Shibuya H, Iinuma H, Shimada R, Horiuchi A and Watanabe $\mathrm{T}$ : Clinicopathological and prognostic value of microRNA-21 and microRNA-155 in colorectal cancer. Oncology 79: 313-320, 2010.

12. Yu F, Yao H, Zhu P, Zhang X, Pan Q, Gong C, Huang Y, Hu X, $\mathrm{Su} F$, Lieberman $\mathrm{J}$ and Song E: let-7 regulates self renewal and tumorigenicity of breast cancer cells. Cell 131: 1109-1123, 2007.

13. Corney DC, Hwang CI, Matoso A, Vogt M, Flesken-Nikitin A, Godwin AK, Kamat AA, Sood AK, Ellenson LH, Hermeking $H$ and Nikitin AY: Frequent downregulation of miR-34 family in human ovarian cancers. Clin Cancer Res 16: 1119-1128, 2010.

14. Wang R, Ma J, Wu Q, Xia J, Miele L, Sarkar FH and Wang Z: Functional role of miR-34 family in human cancer. Curr Drug Targets 14: 1185-1191, 2013.

15. Akao Y, Nakagawa Y and Naoe T: let-7 microRNA functions as a potential growth suppressor in human colon cancer cells. Biol Pharm Bull 29: 903-906, 2006.

16. Esquela-Kerscher A and Slack FJ: Oncomirs - microRNAs with a role in cancer. Nat Rev Cancer 6: 259-269, 2006.
17. Lu J, Getz G, Miska EA, Alvarez-Saavedra E, Lamb J, Peck D, Sweet-Cordero A, Ebert BL, Mak RH, Ferrando AA, et al: MicroRNA expression profiles classify human cancers. Nature 435: 834-838, 2005.

18. Volinia S, Calin GA, Liu CG, Ambs S, Cimmino A, Petrocca F Visone R, Iorio M, Roldo C, Ferracin M, et al: A microRNA expression signature of human solid tumors defines cancer gene targets. Proc Natl Acad Sci USA 103: 2257-2261, 2006.

19. Iorio MV and Croce CM: microRNA involvement in human cancer. Carcinogenesis 33: 1126-1133, 2012.

20. Iorio MV, Ferracin M, Liu C-G, Veronese A, Spizzo R, Sabbioni S, Magri E, Pedriali M, Fabbri M, Campiglio M, et al: MicroRNA gene expression deregulation in human breast cancer. Cancer Res 65: 7065-7070, 2005.

21. Lei Q, Shen F, Wu J, Zhang W, Wang J and Zhang L: MiR-101, downregulated in retinoblastoma, functions as a tumor suppressor in human retinoblastoma cells by targeting EZH2. Oncol Rep 32: 261-269, 2014.

22. Wei X, Xiang T, Ren G, Tan C, Liu R, Xu X and Wu Z: miR-101 is down-regulated by the hepatitis $\mathrm{B}$ virus $\mathrm{x}$ protein and induces aberrant DNA methylation by targeting DNA methyltransferase 3A. Cell Signal 25: 439-446, 2013.

23. Luo C, Merz PR, Chen Y, Dickes E, Pscherer A, Schadendorf D and Eichmüller SB: MiR-101 inhibits melanoma cell invasion and proliferation by targeting MITF and EZH2. Cancer Lett 341: 240-247, 2013.

24. Popov VM, Wu K, Zhou J, Powell MJ, Mardon G, Wang C and Pestell RG: The Dachshund gene in development and hormoneresponsive tumorigenesis. Trends Endocrinol Metab 21: 41-49, 2010.

25. Voas MG and Rebay I: Signal integration during development: Insights from the Drosophila eye. Dev Dyn 229: 162-175, 2004.

26. Zhang L, Yang N, Huang J, Buckanovich RJ, Liang S, Barchetti A, Vezzani C, O'Brien-Jenkins A, Wang J, Ward MR, et al: Transcriptional coactivator Drosophila eyes absent homologue 2 is up-regulated in epithelial ovarian cancer and promotes tumor growth. Cancer Res 65: 925-932, 2005.

27. Li Z, Hu J, Sun Y, Li S, Pestell RG and Wu K: EYA promotes proliferation through up-regulation of cyclin D1. Cancer Res 71 (Suppl 8): abs. 2942 Proceedings: AACR 102nd Annual Meeting 2011, April 2-6, 2011; http://cancerres.aacrjournals.org/ content/71/8_Supplement/2942.short.

28. Pignoni F, Hu B, Zavitz KH, Xiao J, Garrity PA and Zipursky SL: The eye-specification proteins So and Eya form a complex and regulate multiple steps in Drosophila eye development. Cell 91: 881-891, 1997.

29. Kumar JP and Moses K: EGF receptor and Notch signaling act upstream of Eyeless/Pax6 to control eye specification. Cell 104: 687-697, 2001.

30. Li C-M, Guo M, Borczuk A, Powell CA, Wei M, Thaker HM, Friedman R, Klein U and Tycko B: Gene expression in Wilms tumor mimics the earliest committed stage in the metanephric mesenchymal-epithelial transition. Am J Pathol 160: 2181-2190, 2002.

31. Wu K, Li Z, Cai S, Tian L, Chen K, Wang J, Hu J, Sun Y, Li X, Ertel A and Pestell RG: EYA1 phosphatase function is essential to drive breast cancer cell proliferation through cyclin D1. Cancer Res 73: 4488-4499, 2013.

32. Sun Y, Kaneko S, Li X and Li X: The PI3K/Akt signal hyperactivates Eyal via the SUMOylation pathway. Oncogene 34: 2527-2537, 2015.

33. Nikpour P, Emadi-Baygi M, Emadi-Andani E and Rahmati S: EYA1 expression in gastric carcinoma and its association with clinicopathological characteristics: A pilot study. Med Oncol 31: 955, 2014

34. Croce CM: Causes and consequences of microRNA dysregulation in cancer. Nat Rev Genet 10: 704-714, 2009.

35. Sassen S, Miska EA and Caldas C: MicroRNA: implications for cancer. Virchows Arch 452: 1-10, 2008.

36. Wang HJ, Ruan HJ, He XJ, Ma YY, Jiang XT, Xia YJ, Ye ZY and Tao HQ: MicroRNA-101 is down-regulated in gastric cancer and involved in cell migration and invasion. Eur J Cancer 46: 2295-2303, 2010.

37. Ambs S, Prueitt RL, Yi M, Hudson RS, Howe TM, Petrocca F, Wallace TA, Liu CG, Volinia S, Calin GA, et al: Genomic profiling of microRNA and messenger RNA reveals deregulated microRNA expression in prostate cancer. Cancer Res 68: 6162-6170, 2008 . 
38. Strillacci A, Valerii MC, Sansone P, Caggiano C, Sgromo A, Vittori L, Fiorentino M, Poggioli G, Rizzello F, Campieri M and Spisni E: Loss of miR-101 expression promotes Wnt/ $\beta$-catenin signalling pathway activation and malignancy in colon cancer cells. J Pathol 229: 379-389, 2013.

39. Smits M, Nilsson J, Mir SE, van der Stoop PM, Hulleman E, Niers JM, de Witt Hamer PC, Marquez VE, Cloos J, Krichevsky AM, et al: miR-101 is down-regulated in glioblastoma resulting in EZH2-induced proliferation, migration, and angiogenesis. Oncotarget 1: 710-720, 2010.

40. Xu L, Beckebaum S, Iacob S, Wu G, Kaiser GM, Radtke A Liu C, Kabar I, Schmidt HH, Zhang X, et al: MicroRNA-101 inhibits human hepatocellular carcinoma progression through EZH2 downregulation and increased cytostatic drug sensitivity J Hepatol 60: 590-598, 2014.

41. Varambally S, Cao Q, Mani RS, Shankar S, Wang X, Ateeq B, Laxman B, Cao X, Jing X, Ramnarayanan K, et al: Genomic loss of microRNA-101 leads to overexpression of histone methyltransferase EZH2 in cancer. Science 322: 1695-1699, 2008.

42. Buechner J, Tømte E, Haug BH, Henriksen JR, Løkke C, Flægstad T and Einvik C: Tumour-suppressor microRNAs let-7 and miR-101 target the proto-oncogene MYCN and inhibit cell proliferation in MYCN-amplified neuroblastoma. Br J Cancer 105: 296-303, 2011.

43. Graziussi DF, Suga H, Schmid V and Gehring WJ: The 'eyes absent' (eya) gene in the eye-bearing hydrozoan jellyfish Cladonema radiatum: conservation of the retinal determination network. J Exp Zoolog B Mol Dev Evol 318: 257-267, 2012.
44. Li X, Wang S and Schor N: Methylase meets phosphatase: roles of PRMT and EYA1 in neuroblastoma. Cancer Res 74 (Suppl 19): abs. 464, 2014

45. Wang J, Cai S, Chen K, Li S, Pestell R and Wu K: Regulation of AR transcriptional activity and prostate cancer cellular proliferation by DACH1/Eya1/Six1 pathway. Cancer Res 73 (Suppl 8): abs. 1319, 2013.

46. Bolós V, Grego-Bessa J and de la Pompa JL: Notch signaling in development and cancer. Endocr Rev 28: 339-363, 2007.

47. Allenspach EJ, Maillard I, Aster JC and Pear WS: Notch signaling in cancer. Cancer Biol Ther 1: 466-476, 2002.

48. Miele L, Golde T and Osborne B: Notch signaling in cancer. Curr Mol Med 6: 905-918, 2006.

49. Wang Z, Li Y, Banerjee S, Kong D, Ahmad A, Nogueira V, Hay N and Sarkar FH: Down-regulation of Notch-1 and Jagged-1 inhibits prostate cancer cell growth, migration and invasion, and induces apoptosis via inactivation of Akt, mTOR, and NF-kappaB signaling pathways. J Cell Biochem 109: 726-736, 2010

50. Kiaris H, Politi K, Grimm LM, Szabolcs M, Fisher P, Efstratiadis A and Artavanis-Tsakonas S: Modulation of notch signaling elicits signature tumors and inhibits hrasl-induced oncogenesis in the mouse mammary epithelium. Am J Pathol 165: 695-705, 2004.

51. Stylianou S, Clarke RB and Brennan K: Aberrant activation of notch signaling in human breast cancer. Cancer Res 66: $1517-1525,2006$ 